\title{
BMJ Open Quality of resistance training description in COPD trials: study protocol for a systematic review
}

\author{
Bennie Westra, ${ }^{1}$ Sander de Wolf, ${ }^{2}$ Eline bij de Vaate, ${ }^{3}$ Monique Legemaat, ${ }^{3}$ \\ André Nyberg, ${ }^{4}$ Peter Klijn ${ }^{3,5}$
}

To cite: Westra B, de Wolf S, bij de Vaate $\mathrm{E}$, et al. Quality of resistance training description in COPD trials: study protocol for a systematic review. BMJ Open 2019;9:e025030. doi:10.1136/ bmjopen-2018-025030

- Prepublication history for this paper is available online. To view these files please visit the journal online (http://dx.do org/10.1136/bmjopen-2018025030).

Received 26 June 2018 Revised 16 0ctober 2018 Accepted 30 November 2018

Check for updates

(c) Author(s) (or their employer(s)) 2019. Re-use permitted under CC BY-NC. No commercial re-use. See rights and permissions. Published by BMJ.

${ }^{1}$ Department of Physiotherapy, Antonius Hospital, Sneek, The Netherlands

${ }^{2}$ Division of Public Health, Academic Medical Centre, University of Amsterdam, Amsterdam, The Netherlands ${ }^{3}$ Department of Pulmonology, Merem Pulmonary Rehabilitation Centre, Hilversum, The

Netherlands

${ }^{4}$ Department of Community Medicine and Rehabilitation, Physiotherapy, Umeå University, Umeå, Sweden

${ }^{5}$ Department of Pulmonology, Amsterdam University Medical Center, Amsterdam, The Netherlands

Correspondence to Dr Peter Klijn; pklijn@merem.nl

\section{ABSTRACT}

Introduction Limb muscle dysfunction is a common manifestation in patients with chronic obstructive pulmonary disease (COPD). Optimising of limb muscle function is therefore an important goal during pulmonary rehabilitation of patients with COPD. Resistance training (RT) is the best available intervention to achieve this goal. Previous systematic reviews on RT primarily focused on methodological quality. However, the intervention holds the essence of each experimental study. Replication of RT interventions requires clear, complete and accessible reporting of the essential components. The American College of Sports Medicine (ACSM) provides evidencebased guidelines for RT prescription and recommends RT models specific to desired outcomes, that is, improvements in strength, muscular hypertrophy, power or local muscle endurance. The aim of this review is to investigate if the application of the RT principles and key training variables is described sufficiently in current evidence on the effects of $\mathrm{RT}$ interventions in patients with COPD.

Methods and analysis Any research study (randomised, non-randomised controlled, controlled pre-post studies and observational studies) with an RT intervention in patients with COPD will be considered for this systematic review. Potentially relevant studies published in English from inception to 1 October 2017 will be identified from Embase, Cochrane Library, Cumulative Index of Nursing and Allied Health Literature (CINAHL) and Physiotherapy Evidence Database (PEDro). Studies exploring the effects of RT following a single session and RT interventions limited to other respiratory chronic diseases will not be included. Additionally, studies including non-COPD participants will be excluded, if the COPD data are not separated. Pairs of reviewers will independently extract data using data collecting sheets. Quality appraisal of RT description will be performed in timeframes according to the latest published ACSM position statement on exercise or RT.

Ethics and dissemination This protocol is a systematic review and therefore ethical approval is not required. The results of this review will be disseminated through peer-reviewed publication and presented at scientific conferences.

PROSPERO registration number CRD42017067403.

\section{INTRODUCTION}

Chronic obstructive pulmonary disease (COPD) is expected to be the third ranked

\section{Strengths and limitations of this study}

This protocol is reported in line with the Preferred Reporting Items for Systematic Reviews and MetaAnalyses Protocols, and the systematic review (SR) will be reported in line with the Preferred Reporting Items for Systematic Reviews and MetaAnalyses Statement.

- The proposed SR will include all English language published descriptions of resistance training (RT) in clinical studies in patients with chronic obstructive pulmonary disease and will provide a broad overview of the quality of RT prescription.

- Exclusion of clinical studies published in other languages than English will leave potentially relevant information not known to the authors out of the review and this may bias the results.

cause of mortality by $2020 .{ }^{1}$ COPD is primarily characterised by airway limitation. Nonetheless, many patients with COPD experience extrapulmonary consequences that may contribute to disease severity in individual patients. Limb muscle dysfunction is an apparent manifestation in the early stages of the disease process. ${ }^{2}$ Recognised features include limb muscle weakness, muscle atrophy, reduction in muscle oxidative capacity (lower fraction of type I fibres, decreased capillary density and aerobic enzymes), type II fibre shift, all of which have prognostic significance independent of the degree of airway limitation and contribute to exercise limitation. ${ }^{3-5}$

The first American Thoracic Society (ATS) publications on the effects of exercise training during pulmonary rehabilitation (PR) in patients with COPD begun to appear in the 1980s. ${ }^{67}$ These prior statements refer to whole-body, aerobic exercise as a means to improve exercise tolerance. Indeed, traditionally, aerobic exercise training has become the mainstay during COPD rehabilitation. This type of exercise training has well-established effects on patients with COPD with 
improvements in quality of life and exercise capacity. ${ }^{89}$ During the next decade, focus was shifted to the role of peripheral muscle weakness, ${ }^{10}$ and its contribution to exercise limitation. ${ }^{10-12}$ However, aerobic exercise training has little effect on muscle strength. ${ }^{913}$ Resistance training (RT), on the other hand, has shown to improve muscle strength in patients with COPD. ${ }^{14}$ Moreover, RT recruits small muscle groups and results in a lower burden on the ventilatory system with less resultant disabling dyspnoea compared with aerobic endurance training. ${ }^{9}{ }^{15}$ However, although it is stated that RT is a valuable strategy to improve muscle strength and muscle mass, the optimal RT prescription for patients with COPD has yet to be determined..$^{916}$

Determination of the most optimal RT prescription in COPD is, however, more complicated as it appears at first sight. COPD limb muscle is characterised by a large heterogeneity of muscle phenotypes and muscle dysfunction. ${ }^{1718}$ This heterogeneity is related to exercise performance. ${ }^{5}$ In advanced COPD, there is a predominant loss of type II muscle fibres which affects muscle mass and strength. ${ }^{10}$ Moreover, COPD is characterised by a selective loss of type I muscle fibres and oxidative enzymes which affects the endurance function of skeletal muscle, ${ }^{19}$ and is already observed in less severe COPD. ${ }^{20}$ Therefore, these specific alterations in muscle function stress the importance of identifying those factors that should be considered in the development of individualised RT programmes in patients with COPD. Since RT allows appropriate and specific loading to limb muscle, it is deemed the most efficacious approach to ameliorate muscle dysfunction (eg, muscle strength and muscle endurance). ${ }^{3821}$

Appropriate recommendations across the spectrum of muscle dysfunction based on results of RT interventions in patients with COPD require consistent and accurate reporting of the principles of RT prescription. Moreover, the components of the RT intervention should be reported with sufficient detail to guarantee intervention implementation and replication. ${ }^{22}$

The evolution of the present knowledge base of RT guidelines dates back to the post-World War II decades. ${ }^{23-32}$ However, it lasted to the second half of the 1980s when the potential value of RT for health promotion and fitness was recognised by medical and scientific bodies. ${ }^{33}$

In 1990, the American College of Sports Medicine (ACSM) replaced their 1978 Position Statement on exercise training and added an RT component. ${ }^{34}$ The ACSM provides evidence-based guidelines for individualised RT prescription in their subsequent published RT recommendations. ${ }^{21} 3536$ Due to the developments in RT research during the last two decades, it is regarded important to consistently incorporate the principles of RT progression: progressive overload, specificity and variation. ${ }^{21}{ }^{36}$ The principles of RT progression are shown and explained in table $1 .{ }^{21}$ Moreover, reporting of the components of the RT intervention should include RT principles and should be done according to identified key training variables. The key RT variables that determine the demands placed on the body and affect muscle function and morphology are shown in table $2 .^{2136}$

\section{Objectives}

The objectives of this systematic review (SR) are (1) to investigate if the application of the RT principles and (2) the reporting of the key programme variables is described sufficiently in current evidence on the effects of RT interventions in COPD.

\section{METHODS}

The protocol for this SR is in line with the Preferred Reporting Items for Systematic Reviews and Meta-Analyses (PRISMA) Protocols. ${ }^{37}$

Table 1 Principles of resistance training (RT) progression

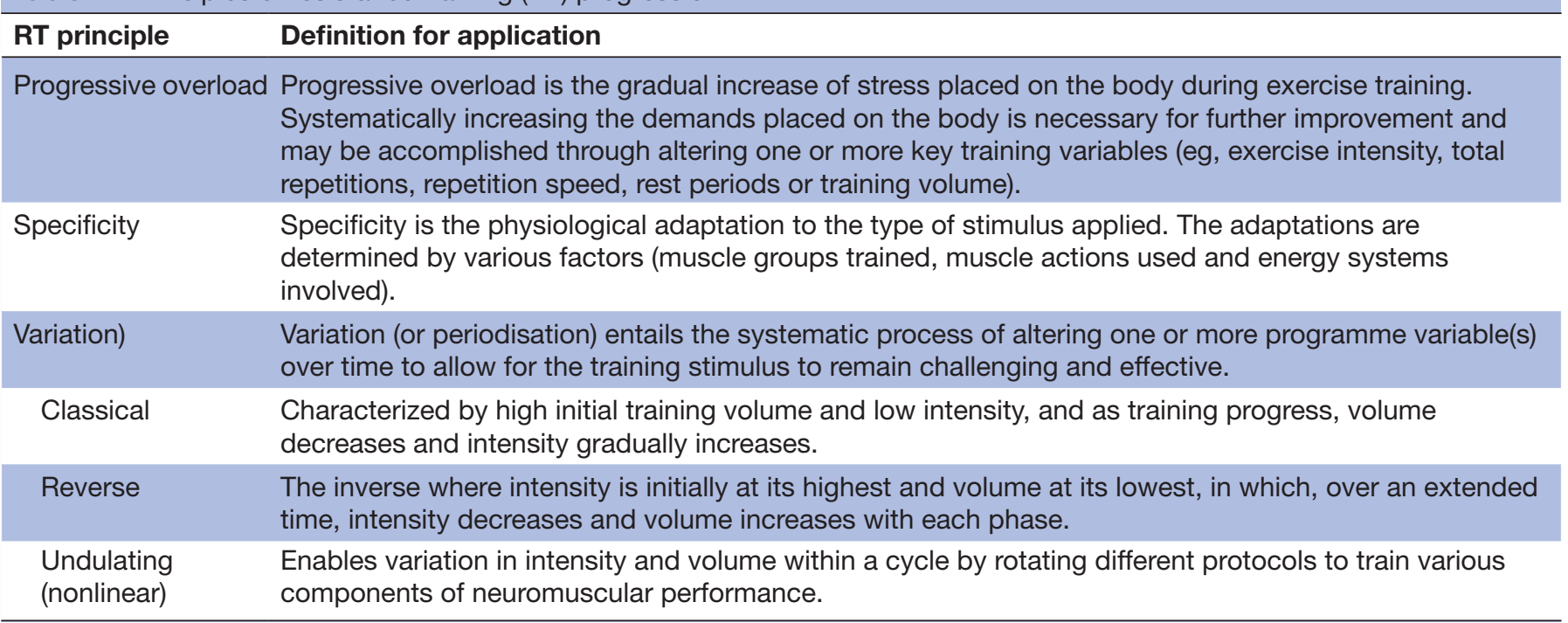


Table 2 Key training variables for resistance training (RT)

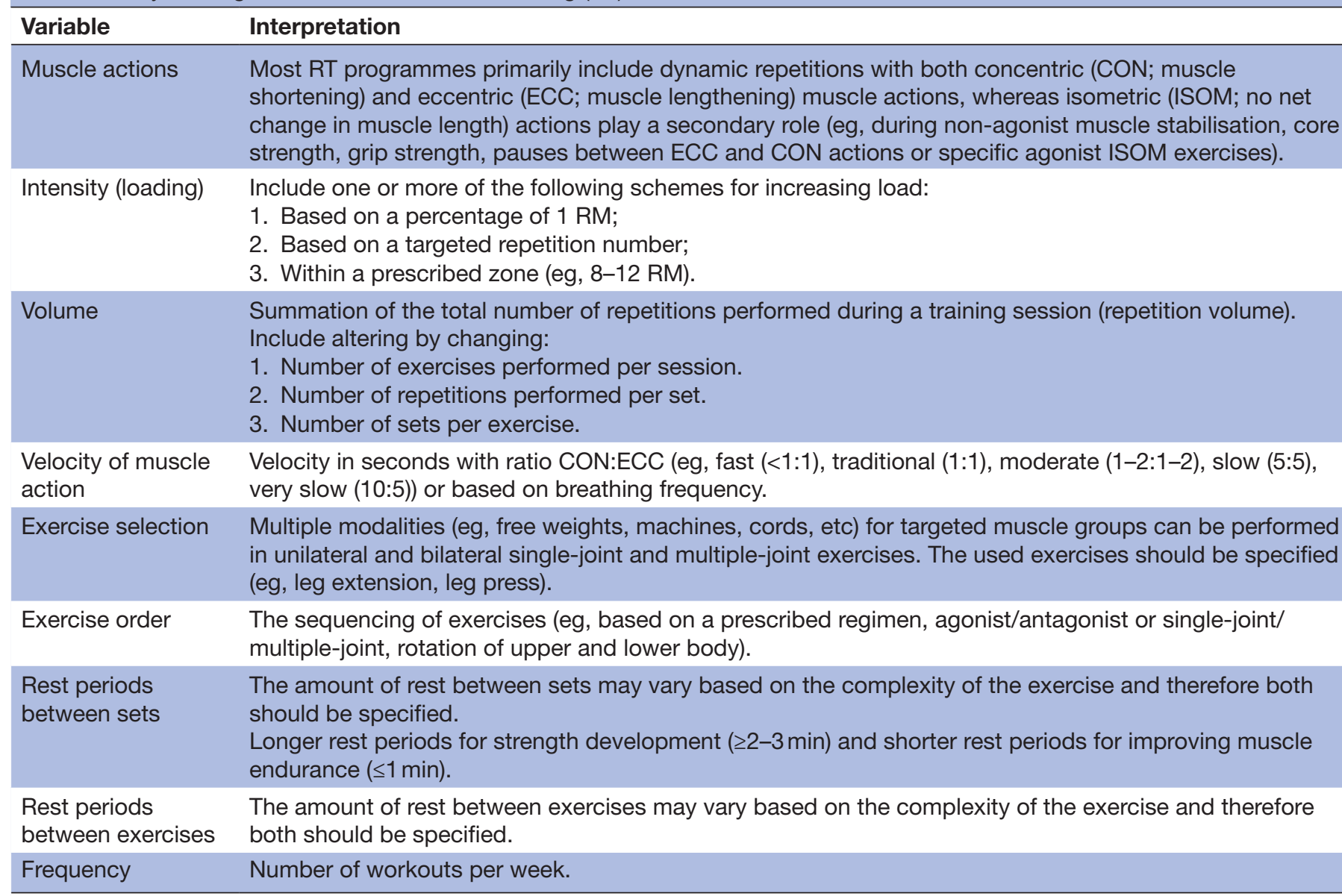

$\mathrm{RM}$, Repetition Maximum.

\section{Inclusion and exclusion criteria}

Any research study (randomised, non-randomised controlled, controlled pre-post studies and observational studies) with an RT intervention in patients with COPD will be considered for this SR. However, studies exploring the effects of RT following a single session and RT interventions limited to other respiratory chronic diseases will not be included. Additionally, studies including non-COPD participants will be excluded, if the COPD data were not separated. No restrictions will be placed on interventions in control groups. Studies referring to a protocol or a secondary study will be taken into account. Only papers published from inception to 1 October 2017 and written in English will be eligible for inclusion. Papers referring to methods described elsewhere will only be included if these references are published in English. The number of non-English publications will however also be reported.

\section{Information sources and search strategy}

To identify relevant studies, a comprehensive search of electronic databases will be performed. The original search strategy will be designed with the assistance of a librarian. The main PubMed/Medline search strategy based on a combination of relevant terms is shown in table 3 . The proposed search strategy terms will be adapted for each electronic database, that is, Ovid (Embase), Cochrane Library Cochrane Controlled Register of Trials (CENTRAL), CINAHL (EBSCO) and PEDro. A manual search of reference lists of included studies and other relevant review studies will also be conducted to obtain additional papers.

\section{Selection process}

The results will be downloaded to Endnote X7 software to manage the records retrieved from the searches, and where duplicates will be removed.

Two reviewers (BW, PK) will independently review all titles and abstract to identify potentially relevant studies for inclusion based on the inclusion criteria. Each study is labelled 'include' or 'exclude' and agreement is examined. Studies will be labelled 'unclear' when inclusion or exclusion of a study could not be based on the screening of the title or abstract. Full-text articles for studies labelled 'unclear' will be independently screened by two reviewers (BW, PK) and subsequently included or excluded. Discrepancies will be resolved by consensus or by arbitration by a third author (SW). The results of the detailed selection process will be reported using the PRISMA flow diagram..$^{38}$ Missing papers will be requested from study authors. 


\begin{tabular}{|c|c|}
\hline Search & Query \\
\hline$\# 1$ & $\begin{array}{l}\text { 'Exercise Therapy’[Mesh] OR resistance training[tiab] OR resistance exercise*[tiab] OR weight bearing exercise } \\
\text { program*[tiab] OR strength training[tiab] OR strengthening program[tiab] OR exercise training[tiab] OR } \\
\text { [exercise[tiab] AND training[tiab]] OR weight-lifting strengthening program*[tiab] OR weight-lifting exercise } \\
\text { program*[tiab] OR weight bearing strengthening program*[tiab] }\end{array}$ \\
\hline \#2 & $\begin{array}{l}\text { 'Pulmonary Disease, Chronic Obstructive'[Mesh] OR chronic obstructive pulmonary disease[tiab] OR COPD[tiab] } \\
\text { OR COAD[tiab] OR chronic obstructive lung disease[tiab] OR chronic obstructive airway disease[tiab] OR chronic } \\
\text { airflow obstruction*[tiab] OR chronic bronchitis[tiab] OR pulmonary emphysema*[tiab] }\end{array}$ \\
\hline \#3 & $\begin{array}{l}\text { 'Clinical Trial'[Publication Type] OR 'Randomized Controlled Trial'[Publication Type] OR 'Random Allocation'[Mesh] } \\
\text { OR 'Cohort Studies'[Mesh] OR 'Case-Control Studies'[Mesh] OR ‘Observational Study'[Publication Type] OR } \\
\text { random*[tiab] OR trial[ti] OR RCT[tiab] OR RCTs[tiab] OR [clinical[tiab] AND trial[tiab]] OR observation*[tiab] OR } \\
\text { cohort[tiab] OR prospective[tiab] OR retrospective[tiab] OR case-control[tiab] }\end{array}$ \\
\hline \#4 & \#1 AND \#2 AND \#3 \\
\hline
\end{tabular}

Mesh, Medical Subject Headings; tiab, title/abstract.

\section{Data extraction}

Data extraction will be performed by one author (first reviewer) and four research and field expert authors (second reviewers). From each included study, the first reviewer and a second reviewer will independently extract data on a constructed form created for this study. Two reviewers will examine agreement, and disagreement will be resolved by consensus.

The following study characteristics will be extracted:

- Publication details: name of first author, publication year.

- Study objective: study design, goal of the study.

- Participants characteristics: number of participants, training status and inclusion criteria when specified: disease severity or Global Initiative for Chronic Obstructive Lung Disease (GOLD) classification, baseline forced expiratory volume in one second $\left(\mathrm{FEV}_{1}\right)$ and/or $\mathrm{FEV}_{1} /$ forced vital capacity, smoking status, comorbidity and/or other relevant inclusion criteria, age range.

- Intervention: description of intervention and control group, description of training device used, adjuncts (eg, administration of oxygen, non-invasive ventilation, Heliox, respiratory muscle training, downhill training, vibrating plates), place of RT in intervention (stand alone, part of PR, adjunct), RT objective, duration, supervision, setting (mixed, inpatient, outpatient, home based).

\begin{tabular}{|c|c|c|c|}
\hline Study & $\begin{array}{l}\text { Progressive } \\
\text { overload }\end{array}$ & Specificity & $\begin{array}{l}\text { Variation } \\
\text { (three types of } \\
\text { periodization) }\end{array}$ \\
\hline
\end{tabular}

$\mathrm{N}$, no; Y, yes.
In addition, primary and secondary study outcome measures will be reported.

\section{Quality appraisal of the exercise prescription}

Data collection sheets are developed to evaluate application RT principles (table 4) and key training variables (table 5). Quality appraisal for each study is performed by the first reviewer and a second reviewer not involved in any of the included studies. Agreement will be examined, and disagreement will be resolved by consensus or a by consultation of a third author. Each RT principle and each training variable will be rated accordingly: yes $(\mathrm{Y})$ 'reported', no (N) 'not reported'. Not applicable (NA) is used for 'exercise order' and 'rest between exercises' when only one RT exercise is used. Both key variables are $\mathrm{NA}$ in this context. ' $\mathrm{Y}$ ' will be given a score of ' 1 ', ' $\mathrm{N}$ ' a score of ' 0 ' and NA will be given no score. Sum scores of RT principles and key training variables per study will be calculated, and corrected for the number of NA used (ie, 7 instead of 9 key training variables). Percentages of studies complying with each RT principle and key training variable will also be calculated.

Published RT interventions should always have integrated the latest scientific developments and insights. Therefore, quality appraisal of RT description will be performed in timeframes according to the latest published ACSM position statement on exercise or RT. ${ }^{21}$ 34-36 39 Moreover, we will search for recommendations of major health organisations and key publications on RT to try to implicate the trends of time in our discussion on quality appraisal.

\section{Patients and public involvement}

Patients and the public were not involved in the design of this SR.

\section{DISCUSSION}

Previous SRs in COPD research were primarily focused on methodological criteria and efficacy. ${ }^{40} 41$ These reviews have shown consistent and clinically important effects, 


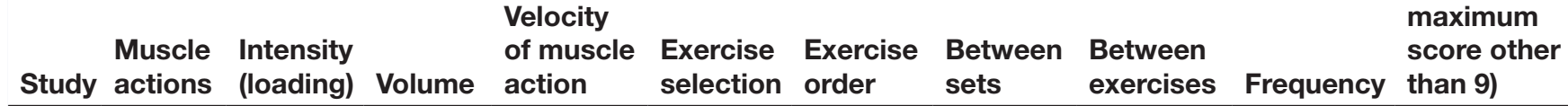

N, no; NA, not applicable; Y, yes.

although no clear RT guidelines could be formulated. Clearly, it is evident that the design and methodological quality of clinical studies are thoroughly and accurately described to allow critical appraisal of study quality. ${ }^{38}$ However, explicit information on the prescribed RT and the actual level of patient compliance by adequate supervision provides the best evidence with respect to the effects of RT interventions. ${ }^{42} 43$ However, it is recognised that inclusion of all intervention information is not always possible due to restrictions in word allowance. ${ }^{44}$ More recent publications might contain more detailed information due to the possibility to publish online supplements or trial protocols. Our findings will be interpreted with consideration of publication opportunities. In order to be able to evaluate the intervention quality, all details of RT interventions should be provided. The chosen RT protocol will have significant impact on study outcome. ${ }^{4546}$ Given the heterogeneity of muscle phenotypes and muscle dysfunction within the COPD population, ${ }^{17} 1845$ a detailed and comprehensive reporting on the RT principles and key training variables is necessary. Detailed information is important for clinicians and allied health professionals to draw valid conclusions, to implement RT interventions for the desired outcome and facilitate reproducibility by other researchers. ${ }^{44}$ Statements on PR and Clinical Practice guidelines are used by clinicians to implement firm evidence-based interventions in clinical practice. Therefore, we will also discuss how RT recommendations are reflected in the official documents of the ATS and the European Thoracic Society, and the formulated practice guidelines.

To our knowledge, this is the first SR with a comprehensive synthesis of existing evidence on the quality of RT description in COPD trails. Our SR has important strengths, since the protocol is reported in line with the PRISMA Protocols, and the SR will be reported in line with the PRISMA Statement. The proposed SR will include all English language published descriptions of RT in clinical studies in patients with COPD and will provide a broad overview of the quality of RT prescription. However, as clinical studies published in English are eligible for inclusion, potentially relevant information from publications in other languages may leave relevant information out of the review. Therefore, the number of non-English publications will be addressed in the discussion on our findings.

\section{CONCLUSION}

The results of this project will provide important information to guide the design of future RT interventions and clinical work in patients with COPD. Reporting of the RT prescription with sufficient detail can produce evidence-based recommendations for a minimum set of key RT variables and improve RT prescription in future PR guidelines.

Acknowledgements The authors would like to thank Faridi van Etten-Jamuldin for assistance with the literature search.

Contributors PK and BW are the guarantors for the protocol and contributed equally to the development of the protocol and they also drafted the protocol. All authors contributed to the development of the eligibility criteria, assessment strategy, search strategy and data extraction criteria. They also read, provided feedback and approved the revised final protocol.

Funding The authors have not declared a specific grant for this research from any funding agency in the public, commercial or not-for-profit sectors.

Competing interests None declared.

Patient consent for publication Not required.

Provenance and peer review Not commissioned; externally peer reviewed.

Open access This is an open access article distributed in accordance with the Creative Commons Attribution Non Commercial (CC BY-NC 4.0) license, which permits others to distribute, remix, adapt, build upon this work non-commercially, and license their derivative works on different terms, provided the original work is properly cited, appropriate credit is given, any changes made indicated, and the use is non-commercial. See: http://creativecommons.org/licenses/by-nc/4.0/.

\section{REFERENCES}

1 Decramer M, Janssens W, Miravitlles M. Chronic obstructive pulmonary disease. Lancet 2012;379:1341-51.

2 Rabe KF, Hurd S, Anzueto A, et al. Global strategy for the diagnosis, management, and prevention of chronic obstructive pulmonary disease: GOLD executive summary. Am J Respir Crit Care Med 2007;176:532-55.

3 Casaburi R. Skeletal muscle function in COPD. Chest 2000;117(5 Suppl 1):267S-71.

4 Jones SE, Maddocks M, Kon SS, et al. Sarcopenia in COPD: prevalence, clinical correlates and response to pulmonary rehabilitation. Thorax 2015;70:213-8.

5 Patel MS, Mohan D, Andersson YM, et al. Phenotypic characteristics associated with reduced short physical performance battery score in COPD. Chest 2014;145:1016-24. 
6 Hodgkin JE, Farrell MJ, Gibson SR, et al. American Thoracic Society. Medical section of the American Lung Association. Pulmonary rehabilitation. Am Rev Respir Dis 1981;124:663-6.

7 American Thoracic Society. Statement on standards for the diagnosis and care of patients with Chronic Obstructive Pulmonary Disease (COPD) and Asthma. Am Rev Respir Dis 1987;136:225-44.

8 Klijn P, van Keimpema A, Legemaat M, et al. Nonlinear exercise training in advanced chronic obstructive pulmonary disease is superior to traditional exercise training. A randomized trial. $\mathrm{Am} \mathrm{J}$ Respir Crit Care Med 2013;188:193-200.

9 Spruit MA, Singh SJ, Garvey C, et al. An official American Thoracic Society/European Respiratory Society statement: key concepts and advances in pulmonary rehabilitation. Am J Respir Crit Care Med 2013;188:e13-e64.

10 Bernard S, LeBlanc P, Whittom F, et al. Peripheral muscle weakness in patients with chronic obstructive pulmonary disease. Am J Respir Crit Care Med 1998;158:629-34.

11 Gosselink R, Troosters T, Decramer M. Peripheral muscle weakness contributes to exercise limitation in COPD. Am J Respir Crit Care Med 1996;153:976-80.

12 Hamilton AL, Killian KJ, Summers E, et al. Symptom intensity and subjective limitation to exercise in patients with cardiorespiratory disorders. Chest 1996;110:1255-63.

13 American Thoracic Society / European Respiratory Society. Skeletal muscle dysfunction in chronic obstructive pulmonary disease. A statement of the American Thoracic Society and European Respiratory Society. Am J Respir Crit Care Med 1999;159(4 Pt 2):S1-40.

14 Simpson K, Killian K, McCartney N, et al. Randomised controlled trial of weightlifting exercise in patients with chronic airflow limitation. Thorax 1992;47:70-5.

15 Lazaar AL, Greenhaff PL. Impaired muscle mitochondrial density and/or function: a COPD-specific mitochondropathy or simply deconditioning? Eur Respir J 2012;40:1070-1.

16 McCarthy B, Casey D, Devane D, et al. Pulmonary rehabilitation for chronic obstructive pulmonary disease. Cochrane Database Syst Rev 2015;2:CD003793.

17 Gouzi F, Abdellaoui A, Molinari N, et al. Fiber atrophy, oxidative stress, and oxidative fiber reduction are the attributes of different phenotypes in chronic obstructive pulmonary disease patients. $J$ Appl Physiol 2013;115:1796-805.

18 Natanek SA, Gosker HR, Slot IG, et al. Heterogeneity of quadriceps muscle phenotype in chronic obstructive pulmonary disease (Copd); implications for stratified medicine? Muscle Nerve 2013;48:488-97.

19 Allaire J, Maltais F, Doyon JF, et al. Peripheral muscle endurance and the oxidative profile of the quadriceps in patients with COPD. Thorax 2004;59:673-8

20 van den Borst B, Slot IG, Hellwig VA, et al. Loss of quadriceps muscle oxidative phenotype and decreased endurance in patients with mild-to-moderate COPD. J Appl Physiol 2013;114:1319-28.

21 American College of Sports Medicine. American College of Sports Medicine position stand. Progression models in resistance training for healthy adults. Med Sci Sports Exerc 2009;41:687-708.

22 Campbell KL, Neil SE, Winters-Stone KM. Review of exercise studies in breast cancer survivors: attention to principles of exercise training. Br J Sports Med 2012;46:909-16.

23 DeLorme TL. Restoration of muscle power by heavy-resistance exercises. J Bone Joint Surg Am 1945;27:645-67.

24 DeLorme TL. Heavy resistance exercises. Arch Phys Med Rehabil 1946;27:607-30.
25 Houtz SJ, Parrish AM, Hellebrandt FA. The influence of heavy resistance exercise on strength. Physiotherap Rev 1946;26:299-304.

26 Delorme TL, Watkins AL. Technics of progressive resistance exercise. Arch Phys Med Rehabil 1948;29:263-73.

27 Zinovieff AN. Heavy-resistance exercises the "Oxford technique". $\mathrm{Br}$ J Phys Med 1951;14:129-32.

28 Macqueen IJ. Recent advances in the technique of progressive resistance exercise. Br Med J 1954;2:1193-8.

29 Hellebrandt FA, Houtz SJ. Mechanisms of muscle training in man: experimental demonstration of the overload principle. Phys Ther Rev 1956;36:371-83.

30 Hellebrandt FA. Application of the overload principle to muscle training in man. Am J Phys Med 1958;37:278-83.

31 Berger R. Effect of varied weight training programs on strength. Res Q 1962;33:168-81.

32 Berger RA. Effect of varied sets of static training on dynamic strength. Am Correct Ther J 1972;26:52-4.

33 Feigenbaum MS, Pollock ML. Prescription of resistance training for health and disease. Med Sci Sports Exerc 1999;31:38-45.

34. American College of Sports Medicine position stand. The recommended quantity and quality of exercise for developing and maintaining cardiorespiratory and muscular fitness in healthy adults. Med Sci Sports Exerc 1990;22:265-74.

35. American College of Sports Medicine Position Stand. The recommended quantity and quality of exercise for developing and maintaining cardiorespiratory and muscular fitness, and flexibility in healthy adults. Med Sci Sports Exerc 1998;30:975-91.

36 Kraemer WJ, Adams K, Cafarelli E, et al. American College of Sports Medicine position stand. Progression models in resistance training for healthy adults. Med Sci Sports Exerc 2002;34:364-80.

37 Moher D, Shamseer L, Clarke M, et al. Preferred reporting items for systematic review and meta-analysis protocols (PRISMA-P) 2015 statement. Syst Rev 2015;4:1.

38 Moher D, Liberati A, Tetzlaff J, et al. Preferred reporting items for systematic reviews and meta-analyses: the PRISMA statement. Int $J$ Surg 2010;8:336-41.

39 American College of Sports Medicine position statement on the recommended quantity and quality of exercise for developing and maintaining fitness in healthy adults. Med Sci Sports 1978;10:vii-x.

40 O'Shea SD, Taylor NF, Paratz J. Peripheral muscle strength training in COPD: a systematic review. Chest 2004;126:903-14.

41 O'Shea SD, Taylor NF, Paratz JD. Progressive resistance exercise improves muscle strength and may improve elements of performance of daily activities for people with COPD: a systematic review. Chest 2009;136:1269-83.

42 Conn VS. The ghost in the machine: identifying mediators of intervention effectiveness. West J Nurs Res 2011;33:867-9.

43 Glasziou P, Meats E, Heneghan C, et al. What is missing from descriptions of treatment in trials and reviews? BMJ 2008;336:1472-4.

44 Abell B, Glasziou P, Hoffmann T. Reporting and replicating trials of exercise-based cardiac rehabilitation: do we know what the researchers actually did? Circ Cardiovasc Qual Outcomes 2015;8:187-94

45 Evans RA, Kaplovitch E, Beauchamp MK, et al. Is quadriceps endurance reduced in COPD?: a systematic review. Chest 2015;147:673-84.

46 Janaudis-Ferreira T, Wadell K, Sundelin G, et al. Thigh muscle strength and endurance in patients with COPD compared with healthy controls. Respir Med 2006;100:1451-7. 